\title{
Moderation Effects of Ethnic-Racial Identity on Disordered Eating and Ethnicity Among Asian and Caucasian Americans
}

\author{
Katrina T. Obleada ${ }^{1 *}$ and Brooke L. Bennett ${ }^{1,2}$ \\ ${ }^{1}$ Department of Psychology, University of Hawai'i at Manoa, Honolulu, HI, United States, ${ }^{2}$ Department of Psychology, Yale \\ University School of Medicine, New Haven, CT, United States
}

Background: The current study was designed to examine whether ethnic-racial identity $(E R I)$ moderated the relationship between disordered eating and primary ethnic identification.

Methods: Three hundred and ninety-eight undergraduate women ( $M_{\text {age }}=19.95$, $S D=3.09$ ) were recruited from a large university in Hawai' $i$. Participants completed the Eating Disorder Examination Questionnaire (EDE-Q), the ERI measure, and reported their

OPEN ACCESS

Edited by:

Paula M. Brochu,

Nova Southeastern University,

United States

Reviewed by:

Chu Shan Elaine Chew,

KK Women's and Children's

Hospital, Singapore

Michael P. Vallario,

VA Palo Alto Health Care System,

United States

${ }^{*}$ Correspondence:

Katrina T. Obleada

kobleada@hawaii.edu

Specialty section:

This article was submitted to

Eating Behavior,

a section of the journal

Frontiers in Psychology

Received: 13 August 2020

Accepted: 22 February 2021

Published: 16 April 2021

Citation:

Obleada KT and Bennett BL (2021)

Moderation Effects of Ethnic-Racial

Identity on Disordered Eating and

Ethnicity Among Asian and Caucasian

Americans.

Front. Psychol. 12:594391.

doi: 10.3389/fpsyg.2021.594391 primary ethnicity as an index of ethnicity.

Results: There was a significant correlation between eating concerns and centrality, $r_{(357)}=0.127, p<0.05$. Moderation analyses indicated that only ERI centrality moderated the predictive effect of ethnicity on the importance of eating concerns, $b=0.05, t_{(347)}=2.37, p=0.018$.

Conclusions: The results suggest that the relationship between self-reported primary ethnicity and EDEQ scores is greater when ethnicity is more central to the individual's identity or when the in-group affect is important to an individual. Findings underscore the need for further research on the underlying mechanisms that account for the differing ways that ERI may affect eating concerns.

Keywords: centrality, weight, shape, ethnic-racial identity exploration, disordered eating and exercise behaviors, in-group affect

\section{INTRODUCTION}

Eating disorders are serious illnesses, characterized by preoccupation with food and overvaluation of weight and shape, that cause severe psychological distress, role impairment, physiological problems, and interpersonal difficulties (Fairburn, 2008; Smink et al., 2012). Anorexia nervosa $(\mathrm{AN})$, bulimia nervosa $(\mathrm{BN})$, and binge-eating disorder (BED) are the three principal eating disorder diagnoses; however, it is also common for individuals to suffer from disordered eating (e.g., restriction, binge-eating, self-induced vomiting, compensatory exercise, and laxative and diuretics misuse) that does not meet the full criteria for these categories (Other Specified Feeding and Eating Disorder: OSFED). In the United States, lifetime prevalence rates for eating disorders are substantial, ranging from 0.6 to $4.5 \%$ and affecting mostly young women; lifetime prevalence rates for OSFED are estimated at $4.78 \%$ in adolescents and $4.64 \%$ in adults (Hudson et al., 2007; Le Grange et al., 2012). Research has shown that these conditions are also a global health issue, although they appear to be more prevalent in Western countries (Hoek, 2016). 
Of particular interest is the dearth of research on their development and maintenance within Asian American populations. According to Nicdao et al. (2007), eating disorders affect Asian Americans at lower prevalence rates than the United States population as a whole. Asian Americans are less likely to seek treatment for psychological problems in general, however, suggesting that prevalence rates may be underestimates (Smart et al., 2011). Eating disorder pathology may also take somewhat different forms within samples of Asian American women (Thomas et al., 2016). Wardle et al. (2006) found that Asian women held stricter standards about achieving the values of thinness compared to other ethnicities. Despite having lower average body mass relative to Caucasian American women, Asian American women report higher levels of body dissatisfaction (Mitz and Kashubeck, 1999; Koff et al., 2001; Yates et al., 2004). Some investigators note that it can be difficult to diagnose using Western criteria within Asian national samples with eating disorders because Asian women tended not to endorse fears of fat or weight gain (Lee et al., 2010; Nakai et al., 2014a).

\section{Risk Factors for Eating Disorders}

Research on risk factors suggests a complex interaction of biological (Striegel-Moore and Bulik, 2007) and genetic vulnerabilities (Bulik et al., 2016), temperamental variables (Lilenfeld, 2011; Wagner and Vitousek, 2019), family dynamics (Striegel-Moore et al., 1986), and general sociocultural pressures (Striegel-Moore et al., 1986; Wertheim and Paxton, 2011). Though these psychosocial risk factors have been studied mostly in Western and White populations, research has suggested similar correlates in other national, racial, and ethnic groups (Keel and Forney, 2013; Pike et al., 2020). According to StriegelMoore and Bulik (2007), "the single best predictor for developing an eating disorder is being female" (p. 182). Adolescence and young adulthood appear to be especially vulnerable times, possibly due to pubertal changes and fat accumulation; this is peak age of onset for clinical eating disorders and is the developmental period, which highest scores are obtained in population-wide studies (Crisp and Kalucy, 1974; Rosenbaum, 1979; Attie and Brooks-Gunn, 1989; Hsu, 1990). Individuals at these life stages experience particular challenges (e.g., pubertyrelated physical changes amid cultural pressures to maintain a prepubertal body type, pursuit of personal and physical autonomy from parents, identity and personality development) that may make them vulnerable to eating problems (Attie and Brooks-Gunn, 1989).

\section{Other Unique Risk Considerations for Asians}

According to Kawamura (2002), risk factors for body image distortion and disordered eating among Japanese women include adherence to a strict and distinct social hierarchy, emotional restraint, need for social approval, and desire to avoid bringing shame on the family. High parental expectations have been identified as a common risk factor, which subsumes desire to gain parental approval; family recognition through achievement in academics, career, and/or appearance; and management of family criticism about weight, eating, and appearance (Peng and Wright,
1994; Pike and Borovoy, 2004; Ting and Hwang, 2007; Isono et al., 2009; Chang et al., 2014; Smart and Tsong, 2014; Tsong and Smart, 2015). In addition, Asian American women may be subjected to sexist and racial stereotypes, such as assumptions that they should be petite or traditionally feminine, or the expectation that they should meet Western standards for physical appearance (e.g., facial features, skin color, height) that are difficult or impossible for Asian women to fulfill (Hall, 1995). Due to the additional pressure from cultural values, Asian American women must navigate traditional Asian values and mainstream American culture, possibly making them more vulnerable to body dissatisfaction and disordered eating (Wonderlich et al., 2007; Tsong and Smart, 2015).

\section{Ethnic Identity and Disordered Eating}

Of particular interest is the relationship between ethnic identity and disordered eating. Previous research has produced mixed findings. Some results indicate a negative association (Henrickson et al., 2010; Stein et al., 2010) or no association between ethnic identity and eating pathology (Yoshimura, 1995; Stark-Wroblewski et al., 2005). Other studies suggest that ethnic identity may serve as a protective factor against thin-ideal internalization and eating pathology (Rhea and Thatcher, 2013; Rakhkovskaya and Warren, 2014).

Ting and Hwang (2007) proposed that intergenerational cultural strain can occur when acculturated daughters misunderstand their parents' expressions of love and care due to culturally normative parenting, such as criticism and shame regarding eating, weight, and appearance. Consistent with this analysis, Lau et al. (2006) found that Asian Americans with higher levels of body dissatisfaction endorsed a stronger identification (i.e., centrality) with traditional Asian values. It is possible that parental expectations regarding appearance may contribute to relationship strain and body dissatisfaction for individuals who strongly identify with traditional Asian values, such as needing parental approval. It is possible that ethnic-racial centrality determines how influential Asian values are on an individual.

The mixed findings on ethnic identity and disordered eating may be attributable to how researchers have defined the construct of acculturation (i.e., extent of assimilation into the dominant culture). Although it is critical to understand the experience of assimilation, assimilation is not exhaustive of the experiences related to ethnic identity, nor of the impact on psychological well-being, such as disordered eating. Previous studies have also not considered how racial identity (i.e., importance of racialized experiences to one's identity) interacts with ethnicity and disordered eating.

\section{Ethnic-Racial Identity}

Ethnic identity is defined as membership in a group with shared cultural traditions, values, and attitudes; racial identity is conceptualized as a membership in a group with shared biological and physical traits, such as skin color (Wilson and Leaper, 2016). Social psychologists have examined racial identity as an important concept that interacts with ethnicity, especially in the United States, coining the term ethnic-racial identity (ERI) 
(Umana-Taylor et al., 2014). It is defined in three ways: (a) acculturation; (b) ethnic identity (e.g., sense of belonging and positivity to a particular ethnic group); and (c) racial identity (e.g., racial regard, racial ideology, racial centrality). Therefore, the concept ERI encompasses both racial and ethnic experiences, which is an extension of previous research that has focused either on ethnic (Phinney, 1992) or racial identity (Sellers et al., 1998). The present study examined ERI, a synthesis of the constructs of ethnic and racial identity that encapsulates individuals' ethnic background and their racialized experiences (Umana-Taylor et al., 2014). The ethnic-racial identification process includes selflabeling (self and other), ethnic-racial knowledge (e.g., cultural practices), and ethnic racial constancy. This identity process includes specific components such as exploration (i.e., ethnic identity search) and centrality (i.e., group membership is part of your individual identity) (Phinney, 1992; Sellers et al., 1998). With this more expanded construct, a clearer understanding of how ethnic identity impacts disordered eating may be explored.

The purpose of the study was to address this gap in the literature by examining how ERI impacts the relationship between ethnic background and body dissatisfaction. It was hypothesized that ERI would be significantly positively correlated with disordered eating. Furthermore, it was anticipated that ERI centrality (i.e., the importance of ERI) would moderate the relationship between ethnic background and disordered eating (i.e., eating, weight, and shape concerns), such that higher centrality scores will strengthen the positive relationship between ethnic background and disordered eating. It was also predicted that conformity pressure (i.e., the degree to which one feels pressure to conform to one's group) would moderate the relationship between ethnic background and disordered eating (i.e., eating, weight, and shape concerns), such that greater pressures to conform will strengthen the positive relationship between ethnic background and disordered eating.

\section{METHODS}

\section{Participants}

Participants were $398\left(M_{\text {age }}=19.95, S D=3.09\right)$ female undergraduates from a large public university in Hawai' $i$ (Obleada and Bennett, 2021). Participants were recruited through introductory psychology courses and offered partial class credit. Based on the ethnicity with which participants primarily identified, the demographic breakdown was as follows: White (22.9\%), Filipino (16.3\%), Japanese (16.0\%), Chinese (8.4\%), Native Hawaiian (6.1\%), Hispanic/Latina (5.6\%), Korean $(3.3$ $\%)$, Other (4.3\%), Other Pacific Islander (2.0\%), Black or African American (1.8\%), Vietnamese (1.5\%), Okinawan (1.3\%), and American Indian or Alaska Native (0.5\%). Additionally, $12.6 \%$ were international students. Notably, in response to a question about ethnic background, $49.1 \%$ of participants endorsed being multiethnic.

\section{Ethnic/Racial Background}

Participants were asked to provide demographic information such as age, gender, primary language, and years of education completed. For ethnicity, participants were first asked to indicate all the ethnicities/races that comprised their background. Second, participants were asked to identify their primary ethnicity/race. Individuals who primarily identified as Asian or Caucasian were specifically investigated.

\section{Disordered Eating}

Disordered eating was measured using the Eating Disorder Examination Questionnaire (EDE-Q; Fairburn and Beglin, 2008). The EDE-Q is a 28 -question survey that provides a comprehensive assessment of eating disorder psychopathology and related behaviors. The EDE-Q is comprised of four scales: Dietary Restraint (e.g., Have you tried to exclude from your diet any foods that you like in order to influence your shape or weight?), Weight Concern (e.g., Have you had a definite fear that you might gain weight?), Shape Concern (e.g., Have you had a definite desire to have a totally flat stomach?), and Eating Concern (e.g., Have you had a definite fear of losing control over eating?). The four subscales were included to recognize nuances in eating disorder concerns; for example, some individuals are dissatisfied with the contours of their body but do not attend closely to what they weigh, while others focus principally on body weight. A total EDE global score is calculated as the mean of the four scales. EDE-Q subscale items are rated on a seven-point forced-choice format (0-6), with higher scores reflecting greater severity or frequency. In a study of 91 male and female college students, internal consistency was higher for women, with Cronbach's alphas $>0.75$ at both time 1 and time 2 (Rose et al., 2013). Among women, the 7-day test-retest reliabilities were generally high for all subscales: Restraint $(r=0.78)$, Eating Concern $(r=0.83)$, Shape Concern $(r=0.87)$, and Weight Concern $(r=0.90)$ (Rose et al., 2013).

\section{Ethnic-Racial Identity}

As cited in Wilson and Leaper's (2016) study, Cameron's (2004) social identity scales (centrality, in-group affect, in-group ties) and Egan and Perry's (2001) gender identity scales (felt typicality, felt conformity pressure) were combined to assess ERI. Building on previous measures that assess the ethnic-identity process, this measure captures felt conformity pressure and distinguishes between in-group affect and in-group ties. Items were modeled after the gender identity scales because they appraised an individual's underlying evaluations of in-group membership (Wilson and Leaper, 2016). Centrality captures the importance of a particular social identity to one's self-concept. In-group affect is how one feels about belonging to a group. In-group ties refer to the extent of connectedness to other group members. Felt typicality captures how representative one feels oneself to be in reference to other group members. Felt conformity pressure is the degree to which one feels pressure to conform to social norms within his or her group. A sample item of the felt typicality subscale includes I have a lot in common with other people within my ethnic/racial group; items are rated on a five-point Likert scale $(1=$ disagree strongly to $5=$ agree strongly). In Wilson and Leaper's (2016) study, internal consistency was moderate for each domain: centrality $(\alpha=0.79)$, in-group affect $(\alpha=0.83)$, in-group ties $(\propto=0.79)$, felt typicality $(\propto=0.75)$, and felt 
TABLE 1 | Means and standard deviations of outcome measures by ethnic-racial identity.

\begin{tabular}{lcc}
\hline & Asian & White/Caucasian \\
& $\boldsymbol{M}(\mathbf{S D})$ & $\boldsymbol{M}(\boldsymbol{S D})$ \\
\hline EDEQ-weight concern & $17.66(8.63)$ & $18.14(8.96)$ \\
EDEQ-shape concern & $3.80(1.73)$ & $3.75(1.83)$ \\
EDEQ-eating concern & $2.18(1.45)$ & $2.34(1.46)$ \\
ERI-centrality & $16.89(7.88)$ & $34.37(7.88)$ \\
ERI-in-group affect & $20.24(7.00)$ & $35.92(9.24)$ \\
\hline
\end{tabular}

$E D E Q$, eating disorder examination questionnaire; ERI, ethnic-racial identity.

conformity pressure $(\alpha=0.84)$ based on a sample of 848 undergraduate participants. Reliability for the combined scales was satisfactory $(r=0.84$; Wilson and Leaper, 2016).

\section{Procedures and Statistical Analyses}

Participants were recruited through the university's SONA (i.e., subject pool management software program) and were given a link to an online survey with an IRB-approved consent form. First, participants were required to review and indicate consent, then fill out a series of computer-based, self-report measures. Upon completion, participants were awarded with research participation credit on SONA. Descriptive analyses and correlations were conducted using the Statistical Package for Social Sciences (SPSS) 26.0. Pearson correlations tested initial associations between all predictor and outcome variables. Using the PROCESS v. 3.3 macro for SPSS, moderation analyses of third variables were conducted (Hayes, 2017).

\section{RESULTS}

\section{Preliminary Analyses}

Caucasian Americans reported higher mean scores on Weight Concern, centrality, and in-group affect compared to Asian Americans; however, when Asian subgroups were examined, it was found that individuals of Vietnamese and Okinawan descent reported the highest mean scores on all outcome measures (see Tables 1, 2, respectively). Pearson correlations revealed that centrality is significantly correlated only with Weight Concern (see Table 3). Consistent with previous literature, Weight Concern was positively associated with centrality $r_{(357)}=0.127$, $p<0.05$. Eating Concern and centrality also had a positive correlation, $r_{(357)}=0.113, p<0.05$. Shape Concern was positively correlated with in-group affect, $r_{(357)}=0.174, p<0.01$. Weight Concern was also positively associated with in-group affect, $r_{(357)}=0.183, p<0.01$. The global EDEQ score and ingroup affect were positively associated, $r_{(357)}=0.147, p<0.01$. The datasets presented in this study can be found in an online repository (Obleada and Bennett, 2021).

\section{Moderation Analyses}

Primary ethnic identification and ERI were used to predict disordered eating. Each predictor was mean centered, and the interaction between primary ethnic identification and ethnic
TABLE 2 | Means and standard deviations of outcome measures by ethnic-racial identity.

\begin{tabular}{llllll}
\hline & EDEQ-WC & EDEQ-SC & EDEQ-EC & Centrality & $\begin{array}{c}\text { In-group } \\
\text { affect }\end{array}$ \\
\hline $\begin{array}{l}\text { Chinese } \\
(n=33)\end{array}$ & $15.52(7.81)$ & $3.38(1.63)$ & $1.75(1.03)$ & $12.36(2.01)$ & $13.79(2.70)$ \\
$\begin{array}{l}\text { Filipino } \\
(n=64)\end{array}$ & $18.61(8.62)$ & $4.01(1.69)$ & $2.35(1.57)$ & $15.61(3.08)$ & $18.95(3.98)$ \\
$\begin{array}{l}\text { Japanese } \\
(n=63)\end{array}$ & $16.52(7.99)$ & $3.59(1.62)$ & $1.97(1.21)$ & $16.98(3.03)$ & $20.50(5.20)$ \\
$\begin{array}{l}\text { Okinawan } \\
(n=5)\end{array}$ & $22.80(12.62)$ & $4.69(2.24)$ & $3.68(2.26)$ & $23.10(7.67)$ & $29.75(5.80)$ \\
$\begin{array}{l}\text { Korean } \\
(n=13)\end{array}$ & $19.69(9.71)$ & $4.07(2.13)$ & $2.57(1.75)$ & $25.23(5.69)$ & $29.85(5.68)$ \\
$\begin{array}{l}\text { Vietnamese } \\
(n=6)\end{array}$ & $22.33(11.33)$ & $4.85(2.09)$ & $2.90(1.95)$ & $31.17(10.82)$ & $38.04(9.90)$ \\
$\begin{array}{l}\text { White } \\
(n=90)\end{array}$ & $18.14(8.96)$ & $3.75(1.83)$ & $2.34(1.46)$ & $34.37(7.88)$ & $35.92(9.24)$ \\
\hline
\end{tabular}

EDEQ-WC, eating disorder examination questionnaire weight concern subscale; EDEQ$S C$, eating disorder examination questionnaire shape concern subscale; EDEQ-EC, eating disorder examination questionnaire eating concern subscale.

TABLE 3 | Pearson's correlations for all outcome measures.

$$
\text { EDEQ-WC EDEQ-SC EDEQ-EC Centrality In-group }
$$

\begin{tabular}{lccccc}
\hline EDEQ-WC & - & - & - & - & - \\
EDEQ-SC & $0.94^{\star \star \star}$ & - & - & - & - \\
EDEQ-EC & $0.73^{\star \star \star}$ & $0.73^{\star \star \star}$ & - & - & - \\
Centrality & 0.08 & 0.04 & $0.15^{\star}$ & - & - \\
In-group affect & $0.13^{\star}$ & 0.10 & $0.15^{\star}$ & $0.80^{\star \star \star}$ & -
\end{tabular}

EDEQ-WC, eating disorder examination questionnaire weight concern subscale; $E D E Q$ $S C$, eating disorder examination questionnaire shape concern subscale; EDEQ-EC, eating disorder examination questionnaire eating concern subscale.

${ }^{*} p<0.05,{ }^{* *} p<0.01,{ }^{* \star *} p<0.001$ (Johnstone, 1987).

racial identity was analyzed using Hayes' PROCESS plug-in for SPSS (Hayes, 2017).

\section{Centrality as a Moderator Between Ethnicity and Eating Concern}

The main effects of primary ethnic identification and centrality were significant predictors of EDEQ Eating Concern $F_{(5,347)}=2.42, p=0.036, R^{2}=0.03$. Both identifying as Asian and identifying as Caucasian were not significantly related to Eating Concern (see Table 4). Eating Concern was not significantly related to centrality of race/ethnicity. Eating Concern was not significantly predicted by the interaction between ethnicity and centrality with identifying as Caucasian. However, Eating Concern was significantly predicted by the interaction between ethnicity and centrality with those identifying as Asian, $b=0.05, t_{(347)}=2.37, p=0.018$. 
TABLE 4 | Linear models of predictors on outcome variables.

\begin{tabular}{|c|c|c|c|c|}
\hline Path & $\boldsymbol{b}$ & df & $t$ & $p$ \\
\hline \multicolumn{5}{|l|}{ Centrality and eating concern } \\
\hline Centrality (M) & 0.01 & 347 & 0.55 & 0.579 \\
\hline Identifying as Asian $\left(X_{1}\right)$ & -0.86 & 347 & -1.54 & 0.124 \\
\hline Identifying as Caucasian $\left(X_{2}\right)$ & -0.28 & 347 & -0.34 & 0.734 \\
\hline Centrality $\times$ identifying as Asian (interaction ${ }_{1}$ ) & 0.05 & 347 & 2.37 & $0.018^{*}$ \\
\hline Centrality $\times$ identifying as Caucasian (interaction ${ }_{2}$ ) & 0.01 & 347 & 0.47 & 0.642 \\
\hline \multicolumn{5}{|l|}{ Centrality and weight concern } \\
\hline Centrality (M) & 0.13 & 345 & 1.66 & 0.098 \\
\hline Identifying as Asian $\left(X_{1}\right)$ & -2.90 & 345 & -0.86 & 0.388 \\
\hline Identifying as Caucasian $\left(X_{2}\right)$ & 6.57 & 345 & 1.36 & 0.176 \\
\hline Centrality $\times$ identifying as Asian (interaction ${ }_{1}$ ) & 0.23 & 345 & 1.68 & 0.095 \\
\hline Centrality $\times$ identifying as Caucasian (interaction ${ }_{2}$ ) & -0.22 & 345 & -1.55 & 0.123 \\
\hline \multicolumn{5}{|l|}{ In-group affect and weight concern } \\
\hline In-group affect (M) & 0.15 & 345 & 2.33 & $0.021^{*}$ \\
\hline Identifying as Asian $\left(X_{1}\right)$ & -1.37 & 345 & -0.41 & 0.683 \\
\hline Identifying as Caucasian $\left(X_{2}\right)$ & 5.19 & 345 & 1.15 & 0.252 \\
\hline Centrality $\times$ identifying as Asian (interaction ${ }_{1}$ ) & -0.16 & 345 & 1.41 & 0.158 \\
\hline Centrality $\times$ identifying as Caucasian (interaction 2 ) & -0.15 & 345 & -1.26 & 0.210 \\
\hline \multicolumn{5}{|l|}{ In-group affect and shape concern } \\
\hline In-group affect (M) & 0.03 & 347 & 2.31 & $0.022^{*}$ \\
\hline Identifying as Asian $\left(X_{1}\right)$ & -0.10 & 347 & -0.15 & 0.878 \\
\hline Identifying as Caucasian $\left(X_{2}\right)$ & 0.86 & 347 & 0.94 & 0.346 \\
\hline Centrality $\times$ identifying as Asian (interaction ${ }_{1}$ ) & 0.03 & 347 & 1.28 & 0.200 \\
\hline Centrality $\times$ identifying as Caucasian (interaction ${ }_{2}$ ) & -0.03 & 347 & -1.05 & 0.292 \\
\hline
\end{tabular}

Centrality and eating concerns model $R^{2}=0.03$; centrality and weight concerns model $R^{2}=0.04$; in-group affect and weight concerns model $R^{2}=0.05$; in-group affect and shape concerns model $R^{2}=0.04$.

${ }^{\star} p<0.05,{ }^{* *} p<0.01,{ }^{* \star *} p<0.001$ (Johnstone, 1987).

\section{Centrality as a Moderator Between Ethnicity and Weight Concern}

The main effects of primary ethnic identification and centrality were significant predictors of EDEQ Weight Concern, $F_{(5,345)}=2.79, p=0.018, R^{2}=0.04$. Identifying as Asian was not significantly related to Weight Concern nor was identifying as Caucasian. Weight Concern was not significantly related to centrality and was not predicted by the interaction between ethnicity and centrality if identifying as Asian or Caucasian (see Table 4).

\section{In-group Affect as a Moderator Between Ethnicity and Weight Concern}

The main effects of primary ethnic identification and ingroup affect were significant predictors of Weight Concern, $F_{(5,345)}=3.68, p=0.003, R^{2}=0.05$. Neither identifying as Asian nor identifying as Caucasian was significantly related to Weight Concern. Weight Concern was significantly related to in-group affect, $b=0.15, t_{(345)}=2.33, p=0.021$. However, the moderation was not significant as Weight Concern was not significantly predicted by the interaction between ethnicity and in-group affect, when identifying as Asian or when identifying as Caucasian (see Table 4).

\section{In-group Affect as a Moderator Between Ethnicity and Shape Concern}

The main effects of primary ethnic identification and ingroup affect were significant predictors of EDEQ Shape Concern, $F_{(5,347)}=3.22, p=0.008, R^{2}=0.04$. Identifying as Asian was not significantly related to Shape Concern nor was identifying as Caucasian. Shape Concern was significantly related to in-group affect, $b=0.03, t_{(347)}=2.31$, $p=0.022$. However, the moderation was not significant as Shape Concern was not significantly predicted by the interaction between ethnicity and in-group affect, if identifying as Asian nor when identifying as Caucasian (see Table 4).

\section{DISCUSSION}

The current study examined the interrelationship between primary ethnic identification, ERI, and disordered eating. Consistent with the first hypothesis, ERI was significantly, positively correlated with disordered eating (i.e., eating, weight, and shape concerns); specifically, as centrality (i.e., importance of a particular social identity to one's self-concept) increased, eating, weight, and shape concerns increased. The second hypothesis was partially supported, such that centrality moderated the effect of primary ethnic identification on eating concerns when identifying as Asian. Interestingly, this finding suggests that for Asian American individuals, the degree to which eating concerns are distressing may be moderated by how important ethnicity is to one's identity. This effect supports previous findings that have identified family eating concerns as a possible risk factor, particularly for Asian Americans (Smart and Tsong, 2014). An examination of existing literature on cultural beliefs in disordered eating among Asian American women has identified specific values related to family recognition through achievement and emotional self-control as significant predictors of disordered eating (Tsong and Smart, 2015). Additionally, Han (2020) found that tensions from pursuing family recognition and intergenerational conflict influenced binge-eating and restriction only when certain psychological needs (e.g., autonomy, competence, connectedness) were not met, thus implying that this phenomenon may be more nuanced.

In addition to possible family influences, centrality of Asian identity related to distress about eating concerns in Asian American women may be due to appearance pressures and body ideals being central to Asian identity. For example, past research found that Asian women compared to women in countries across Europe, the Mediterranean, and South America held stricter standards about achieving the values of thinness compared to other ethnicities (Wardle et al., 2006). It is possible that individuals who find their Asian race more central to their overall identity could be more likely to subscribe to these values about achieving thinness. Previous findings have also shown 
that some Asian values, particularly conformity to group norms and family recognition through achievement, are associated with disordered eating among Asian American women (Tsong and Smart, 2015; Han, 2020). According to symbolic interaction theory, individuals develop their self-concept based on reflected appraisals (i.e., beliefs about how they are perceived by others), such that their identity is partly construed by perceptions or evaluations that others have of them (Alvarez and Helms, 2001; Aksan et al., 2009). This theory may help to explain why Asian cultures that value conforming to group norms find it especially important to adhere to rules of conductbecause it is integrated into their identity. For Asian culture's collectivistic values of family recognition through achievement and conforming to group norms, symbolic interaction helps to explain the importance that cultural values may play in Asian identity. Therefore, if an Asian American individual highly appraises the values of their family's culture of origin, then it may be important for their self-concept to adhere to these ideals including standards about beauty.

Additionally, though in-group affect was not identified as a moderator, the results suggest that greater in-group affect predicted both greater Shape Concern and greater Weight Concern regardless of one's primarily identified ethnicity. Of particular note is how results demonstrated that culture is important to the Caucasian Americans sampled in this study. It is not clear why in-group affect would be important to these individuals; however, specific cultural aspects related to family country of origin (e.g., Italian, Jewish ancestry) were not assessed and merit further investigation into aspects of eating related to the connectedness of culture. Since almost $50 \%$ of the sample identified as being multiethnic, some Caucasian Americans may also have a multiethnic background that possibly contributed to their culture's influence on eating concerns.

\section{LIMITATIONS AND FUTURE DIRECTIONS}

The first limitation of this study is that it collapsed all Asians ethnicities into one group of Asian Americans for analyses. The demographic breakdown of our participants suggests that the individuals who identified as Asian primarily come from East Asian backgrounds. Therefore, the outcomes are likely more reflective of the experience of East Asian Americans, rather than all Asian Americans. Future research should examine differences in the relationships between ethnic centrality and disordered eating variables across different countries of origin across Asia and Europe. Additionally, research has shown that there are particular nuances of disordered eating concerns within all Asian ethnicities (Lee et al., 1989, 2010; Edman and Yates, 2005; Jackson et al., 2006; Nakai et al., 2014a). Future research should examine underlying mechanisms that account for the influences of ERI on eating concerns and examine when ethnic populations are more affected by this moderation relationship. Another limitation is that the paucity of research within this population could contribute to measurement inaccuracies. While the measures used in the present study had adequate internal consistency and are considered to be valid and reliable more broadly, their psychometric properties have not been examined in a sample with similar ethnic and racial diversity. For example, in the present study, we used a standard measurement tool for assessing eating behaviors, the EDE-Q; however, past research suggests that norms may differ across racial groups (Nakai et al., 2014b). Future research should examine whether this measure is the best fit for assessing eating pathology in diverse samples. Additionally, it may be important for clinicians and researchers to consider how the importance of ethnic identity and its manifestation within disordered eating may affect particular Asian American subgroups, such that understanding these differences may be especially important in informing culturally sensitive treatment and prevention approaches for eating pathology. Given that the results from this study include a multiethnic sample, subsequent research examining the differences between multiethnic and single ethnic identities within the context of eating pathology is also needed. Finally, further research should explore how other aspects of culture may contribute to the value or importance of eating concerns among Caucasian individuals.

\section{CONCLUSIONS}

This study is one of the first to examine centrality within the context of disordered eating concerns. Of the ERI subscales, only centrality had a moderation effect on eating concerns; however, it may also be important to explore how centrality may impact other eating disorder constructs that have been found to be influenced by race/ethnicity, such as body surveillance or drive for thinness. It may also be desirable for clinicians and researchers to consider how the importance of ethnic identity and its manifestation within disordered eating may affect particular ethnic groups. In this study, the effect was more pronounced for Asian Americans compared to Caucasian Americans. Lastly, while the results provide initial evidence that ethnic centrality is potentially an important factor in explaining some of the notable differences in disordered eating pathology between American women who identify as Asian vs. Caucasian, the findings of the present study truly underscore the need for further research across racial and ethnic groups in order to better understand the nuanced differences in experiences of cultural ideals and disordered eating.

\section{DATA AVAILABILITY STATEMENT}

The names of the repository/repositories and accession number(s) can be found below: https://doi.org/10.6084/m9. figshare.12800066.v1.

\section{ETHICS STATEMENT}

The studies involving human participants were reviewed and approved by University of Hawaii at Manoa Human Studies Program. The patients/participants provided their written informed consent to participate in this study. 


\section{AUTHOR CONTRIBUTIONS}

KO contributed to conception and design of the study and organized the database. $\mathrm{KO}$ and $\mathrm{BB}$ performed the statistical analysis. $\mathrm{KO}$ wrote the first draft of the manuscript. Both authors contributed to manuscript revision, read, and approved the submitted version.

\section{REFERENCES}

Aksan, N., Kisac, B., Aydin, M., and Demirbuken, S. (2009). Symbolic interaction theory. Proc. Soc. Behav. Sci. 1, 902-904. doi: 10.1016/j.sbspro.2009.01.160

Alvarez, A., and Helms, J. (2001). Racial identity and reflected appraisals as influences on Asian Americans' racial adjustment. Cult. Divers. Ethnic Minor. Psychol. 7, 217-231. doi: 10.1037/1099-9809.7.3.217

Attie, I., and Brooks-Gunn, J. (1989). Development of eating problems in adolescent girls: a longitudinal study. Dev. Psychol. 25, 70-79. doi: 10.1037/0012-1649.25.1.70

Bulik, C. M., Kleiman, S. C., and Yilmaz, Z. (2016). Genetic epidemiology of eating disorders. Curr. Opin. Psychiatry 29, 383-388. doi: $10.1097 /$ yco.0000000000000275

Cameron, J. E. (2004). A three-factor model of social identity. Self Identity 3, 239-262. doi: 10.1080/13576500444000047

Chang, E. C., Yu, E. A., and Lin, E. Y. (2014). An examination of ethnic variations in perfectionism and interpersonal influences as predictors of eating disturbances: a look at Asian and European American females. Asian Am. J. Psychol. 5, 243-251. doi: 10.1037/a0034621

Crisp, A. H., and Kalucy, R. S. (1974). Aspects of perceptual disorder in anorexia nervosa. Psychol. Psychother. 47, 349-361. doi: 10.1111/j.2044-8341.1974.tb02300.x

Edman, J. L., and Yates, A. (2005). A cross-cultural study of disordered eating attitudes among Filipino and Caucasian Americans. Eat. Disord. 13, 279-289. doi: 10.1080/10640260590932887

Egan, S. K., and Perry, D. G. (2001). Gender identity: a multidimensional analysis with implications for psychosocial adjustment. Dev. Psychol. 37, 451-463. doi: 10.1037//0012-I649.37.4.45I

Fairburn, C., and Beglin, S. (2008). "Eating disorder examination," in Cognitive Behavior Therapy and Eating Disorders, ed C. Fairburn (New York, NY: Guilford Press), 265-308.

Fairburn, C. G. (2008). Cognitive Behavior Therapy and Eating Disorders. New York, NY: Guilford Press.

Hall, C. C. I. (1995). Asian eyes: body image and eating disorders of Asian and Asian American women. Eat. Disord. 3, 8-19. doi: 10.1080/10640269508249141

Han, S. (2020). Asian values, intergenerational conflict, needs, and attachment in Asian/Asian American women's disordered eating. Couns. Psychol. 48, 526-550. doi: $10.1177 / 0011000020903561$

Hayes, A. F. (2017). Introduction to Mediation, Moderation, and Conditional Process Analysis: A Regression-Based Approach. New York, NY: Guilford.

Henrickson, H. C., Crowther, J. H., and Harrington, E. F. (2010). Ethnic identity and maladaptive eating: expectancies about eating and thinness in African American women. Cult. Divers. Ethnic Minor. Psychol. 16, 87-93. doi: $10.1037 / \mathrm{a} 0013455$

Hoek, H. W. (2016). Review of the worldwide epidemiology of eating disorders. Curr. Opin. Psychiatry 29, 336-339. doi: 10.1097/yco.0000000000000282

Hsu, L. K. G. (1990). Eating Disorders. New York, NY: Guilford Press.

Hudson, J. I., Hiripi, E., Pope, H. G., and Kessler, R. C. (2007). The prevalence and correlates of eating disorders in the National Comorbidity Survey Replication. Biol. Psychiatry 61, 348-358. doi: 10.1016/j.biopsych.2006.03.040

Isono, M., Watkins, P. L., and Lian, L. E. (2009). "Bon bon fatty girl: a qualitative exploration of weight bias in Singapore," in The Fat Studies Reader, eds E. Rothblum and S. Solovay (New York, NY: New York University Press), 127-138.

Jackson, S. C., Keel, P. K., and Ho Lee, Y. (2006). Trans-cultural comparison of disordered eating in Korean women. Int. J. Eat. Disord. 39, 498-502. doi: 10.1002/eat.20270

\section{ACKNOWLEDGMENTS}

First, we would like to thank Dr. Kelly Vitousek for her constant guidance and support. Secondly, we would like to express our gratitude to our colleagues, Dr. Allison Wagner, Dr. Kaitlin Hill, and Rachel Marshall, for their input and statistical assistance.

Johnstone, D. J. (1987). Tests of significance following R. A. Fisher. Br. J. Philos. Sci. 38, 481-499. doi: 10.1093/bjps/38.4.481

Kawamura, K. Y. (2002). "Asian American body images," in Body Image: A Handbook of Theory, Research, and Clinical Practice, eds T. F. Cash and T. Pruzinsky (New York, NY: Guilford Press), 243-249.

Keel, P. K., and Forney, K. J. (2013). Psychosocial risk factors for eating disorders. Int. J. Eat. Disord. 46, 433-439. doi: 10.1002/eat.22094

Koff, E., Benavage, A., and Wong, B. (2001). Body-image attitudes and psychological functioning in Euro-American and Asian-American college women. Psychol. Rep. 88, 917-928. doi: 10.2466/pr0.2001.88.3.917

Lau, A. S., Lum, S. K., Chronister, K. M., and Forrest, L. (2006). Asian American college women's body image: a pilot study. Cult. Divers. Ethnic Minor. Psychol. 12, 259-724. doi: 10.1037/1099-9809.12.2.259

Le Grange, D., Swanson, S. A., Crow, S. J., and Merikangas, K. R. (2012). Eating disorder not otherwise specified presentation in the US population. Int. J. Eat. Disord. 45, 711-718. doi: 10.1002/eat.22006

Lee, S., Chiu, H. F., and Chen, C. N. (1989). Anorexia nervosa in Hong Kong. Why not more in Chinese? Br. J. Psychiatry 154, 683-688. doi: 10.1192/bjp.154. 5.683

Lee, S., Ng, K. L., Kwok, K., and Fung, C. (2010). The changing profile of eating disorders at a tertiary psychiatric clinic in Hong Kong. Int. J. Eat. Disord. 43, 307-314. doi: 10.1002/eat.20686

Lilenfeld, L. (2011). "Personality and temperament in eating disorders," in Current Topics in Behavioral Neurosciences, Vol. 6, eds W. H. Kaye and R. Adan (New York, NY: Springer), 3-9.

Mitz, L. B., and Kashubeck, S. (1999). Body image and disordered eating among Asian American and Caucasian college students: an examination of race and gender differences. Psychol. Women Q. 23, 781-796. doi: 10.1111/j.1471-6402.1999.tb00397.x

Nakai, Y., Nin, K., Fukushima, M., Nakamura, K., Noma, S., Teramukai, S., et al. (2014a). Eating disorder examination questionnaire (EDE-Q): norms for undergraduate Japanese women. Eur. Eat. Disord. Rev. 22, 439-442. doi: $10.1002 /$ erv. 2324

Nakai, Y., Nin, K., Teramukai, S., Taniguchi, A., Fukushima, M., and Wonderlich, S. A. (2014b). Typical and atypical anorexia nervosa in a Japanese sample. Int. J. Eat. Disord. 47, 130-137. doi: 10.1002/eat.22208

Nicdao, E. G., Hong, S., and Takeuchi, D. T. (2007). Prevalence and correlates of eating disorders among Asian Americans: results from the national Latino and Asian American study. Int. J. Eat. Disord. 40, S22-S26. doi: 10.1002/eat. 20450

Obleada, K., and Bennett, B. (2021). ERI, Eating, and Ethnicity.xlsx (Version 1) [Data set]. Figshare. doi: 10.6084/m9.figshare.12800066.v1

Peng, S. S., and Wright, D. (1994). Explanation of academic achievement of Asian American students. J. Educ. Res. 87, 346-352. doi: 10.1080/00220671.1994.9941265

Phinney, J. (1992). The multigroup ethnic identity measure: a new scale for use with diverse groups. J. Adolesc. Res. 7, 156-176. doi: 10.1177/074355489272003

Pike, K. M., and Borovoy, A. (2004). The rise of eating disorders in Japan: issues of culture and limitations of the model of "Westernaization." Cult. Med. Psychiatry 28, 493-531. doi: 10.1007/s11013-004-1066-6

Pike, K. M., So, M., Hilbert, A., Maekawa, H., Shimanouchi, T., Wilfley, D., et al. (2020). Risk factors for anorexia nervosa and bulimia nervosa in Japan and compared to a US sample. Int. J. Eat. Disord. 54, 155-167. doi: $10.1002 /$ eat.23442

Rakhkovskaya, L. M., and Warren, C. W. (2014). Ethnic identity, thin-ideal internalization, and eating pathology in ethnically diverse college women. Body Image 11, 438-445. doi: 10.1016/j.bodyim.2014.07.003 
Rhea, D. J., and Thatcher, W. (2013). Ethnicity, ethnic identity, self-esteem, and at-risk eating disordered behavior differences of urban adolescent females. Eat. Disord. 21, 223-237. doi: 10.1080/10640266.2013.779177

Rose, J. S., Vaewsorn, A., Rosselli-Navarra, F., Wilson, G. T., and Weissman, R. S. (2013). Test-retest reliability of the eating disorder examination-questionnaire (EDE-Q) in a college sample. J. Eat. Disord. 1:42. doi: 10.1186/2050-2974-1-42

Rosenbaum, M. B. (1979). "The changing body image of the adolescent girl," in Female Adolescent Development, ed M. Sugar (New York, NY: Brunner/Mazel Publishers), 234-253.

Sellers, R. M., Smith, M. A., Shelton, J. N., Rowley, S. A. J., and Chavous, T. M. (1998). Multidimensional model of racial identity: a reconceptualization of African American racial identity. Person. Soc. Psychol. Rev. 2, 18-39. doi: 10.1207/s15327957pspr0201_2

Smart, R., and Tsong, Y. (2014). Weight, body dissatisfaction, and disordered eating: Asian American women's perspectives. Asian Am. J. Psychol. 5, 344-352. doi: $10.1037 / \mathrm{a} 0035599$

Smart, R., Tsong, Y., Mejia, O., Hayashino, D., and Braaten, M. E. (2011). Therapists' experiences treating Asian American women with eating disorders. Profess. Psychol. 42, 308-315. doi: 10.1037/a0024179

Smink, F. R., van Hoeken, D., and Hoek, H. W. (2012). Epidemiology of eating disorders: incidence, prevalence, and mortality rates. Curr. Psychiatry Rep. 4, 406-414. doi: 10.1007/s11920-012-0282-y

Stark-Wroblewski, K., Yanico, B. J., and Lupe, S. (2005). Acculturation, internalization of Western appearance norms, and eating pathology among Japanese and Chinese international student women. Psychol. Women Q. 29, 38-46. doi: 10.1111/j.1471-6402.2005.00166.x

Stein, K., Corte, C., and Ronis, D. L. (2010). Personal identities and disordered eating behaviors in Mexican American women. Eat. Behav. 11, 197-200. doi: 10.1016/j.eatbeh.2010.02.001

Striegel-Moore, R. H., and Bulik, C. M. (2007). Risk factors for eating disorders. Am. Psychol. 62, 181-198. doi: 10.1037/0003-066x.62.3.181

Striegel-Moore, R. H., Silberstein, L. R., and Rodin, J. (1986). Toward an understanding of risk factors for bulimia. Am. Psychol. 41, 246-263. doi: 10.1037/0003-066x.41.3.246

Thomas, J. J., Lee, S., and Becker, A. E. (2016). Updates in the epidemiology of eating disorder in Asia and the Pacific. Curr. Opin. Psychiatry 29, 354-362. doi: 10.1097/yco.0000000000000288

Ting, J. Y., and Hwang, W. (2007). Eating disorders in Asian American women: integrating multiculturalism and feminism. Women Ther. 30, 145-160. doi: 10.1300/j015v30n04_11
Tsong, Y., and Smart, R. (2015). The role of cultural beliefs in disordered eating among Asian-American women. Asian Am. J. Psychol. 6, 342-349. doi: 10.1037/aap0000029

Umana-Taylor, A. J., Quintana, S. M., Lee, R. M., Cross, W. E. Jr., Rivas-Drake, D., Schwartz, S. J., et al. (2014). Ethnic and racial identity during adolescence and into young adulthood: an integrated conceptualization. Child Dev. 85, 21-39. doi: $10.1111 /$ cdev.12196

Wagner, A. F., and Vitousek, K. M. (2019). Personality variables and eating pathology. Psychiatr. Clin. North Am. 42, 105-119, doi: 10.1016/j.psc.2018.10.012

Wardle, J., Haase, A. M., and Steptoe, A. (2006). Body image and weight control in young adults: international comparisons in university students from 22 countries. Int. J. Obes. 30, 644-651. doi: 10.1038/sj.ijo.0803050

Wertheim, E. H., and Paxton, S. J. (2011). "Body image in adolescent girls," in Body image: A Handbook of Science, Practice and Prevention, eds eds T. F. Cash and L. Smolak (New York, NY: Guilford Press), 76-84.

Wilson, A. R., and Leaper, C. (2016). Bridging multidimensional models of ethnicracial and gender identity among ethnically diverse emerging adults. J. Youth Adolesc. 45, 1614-1637. doi: 10.1007/s10964-015-0323-Z

Wonderlich, S. A., Joiner, T. E., Williamson, D. A., and Crosby, R. D. (2007). Eating disorder diagnoses: empirical approaches to classification, Am. Psychol. 62, 167-180. doi: 10.1037/0003-066x.62.3.167

Yates, A., Edman, J., and Arugete, M. (2004). Ethnic differences in BMI and body/self-dissatisfaction among Whites, Asian subgroups, Pacific Islanders, and African Americans. J. Adolesc. Health 34, 300-307. doi: 10.1016/s1054-139x(03)00305-7

Yoshimura, K. (1995). Acculturation and sociocultural influences on the development of eating disorders in Asian American females. Eat. Disord. 3, 216-228. doi: 10.1080/10640269508249165

Conflict of Interest: The authors declare that the research was conducted in the absence of any commercial or financial relationships that could be construed as a potential conflict of interest.

Copyright (C) 2021 Obleada and Bennett. This is an open-access article distributed under the terms of the Creative Commons Attribution License (CC BY). The use, distribution or reproduction in other forums is permitted, provided the original author(s) and the copyright owner(s) are credited and that the original publication in this journal is cited, in accordance with accepted academic practice. No use, distribution or reproduction is permitted which does not comply with these terms. 